\title{
PRINCIPALES COMPETENCIAS PROFESIONALES REQUERIDAS AL CONTADOR PÚBLICO COLEGIADO POR LAS MICRO Y PEQUEÑAS EMPRESAS DEL PERÚ
}

\author{
KEY SKILLS REQUIRED TO COLLEGE PUBLIC ACCOUNTANT FOR MICRO AND \\ SMALL ENTERPRISES OF PERU
}

\author{
Adrian Alejandro Flores Konja* \\ Docente Principal de la Facultad de Ciencias Contables \\ Manuel Alberto Hidalgo Tupia** \\ Docente Auxiliar de la Facultad de Ciencias Contables \\ Universidad Nacional Mayor de San Marcos-UNMSM / Lima-Perú
}

[Recepción: Mayo de 2013/ Conformidad: Junio de 2013]

\section{RESUMEN}

Se propone determinar cuáles son las competencias profesionales requeridas por las pymes peruanas a los profesionales de la Contabilidad, y otros factores relacionados al campo laboral profesional de los contadores públicos colegiados. Para ello, en base a las competencias profesionales definidas en el Proyecto Educativo de nuestra Facultad de Ciencias Contables de la Universidad decana de América, las contenidas en la Ley 28951, Ley de Profesionalización del Contador Público, y la propuesta en Tuning América, como patrón comparativo, se han formulado cuestionarios de encuesta aplicados a los máximos dirigentes de los gremios de pymes representativos -adscritos a la Sociedad Nacional de Industrias, Asociación de Exportadores, Confederación Nacional de Comerciantes, y los emporios emergentes de Gamarra y Villa El Salvador-para identificar cuáles son las competencias más requeridas por estas al CPC, su perfil personal, los campos de acción dentro de la empresa, las funciones empresariales, y su requerimiento (demanda) relativo al de otras profesiones, como administradores, ingenieros, economistas y abogados.

Las competencias ligadas a la función tributaria son las más requeridas al nivel de las micro y pequeñas empresas; la profesión contable tiene una demanda actual y potencial asegurada en el campo de las mype, $y$ continúa siendo una de las más requeridas entre todas las profesiones por las micro y pequeña empresas.

Palabras clave: contabilidad, competencias profesionales, Competencias formativas, Empresa, Mercado laboral profesional del Contador Público Colegiado, Tributación, Auditoría, Finanzas

\begin{abstract}
It is proposed to identify the skills required by the Peruvian SMEs to accounting professionals, and other factors related to the professional work force of Chartered Certified Accountants. To do this, based on professional competencies defined in the Educational Project of our School of Accounting at the University Dean of America, contained in Law 28951 - Professionalization Act of CPA, and the proposal Tuning America as pattern comparison, there have been survey questionnaires applied to the top leaders of the unions representing SMEs - affiliated to the National Society of Industries Exporters Association, National Retail Federation, and the emerging empires Gamarra and Villa El Salvador - to identify what are the most needed skills for these to CPC, your personal profile, the fields of action within the enterprise, business functions, and its requirement (demand) relative to other professions, such as managers, engineers, economists and lawyers. In a brief analysis of the results, it has come to the conclusions that: competencies linked to the tax function are most needed at the level of micro and small enterprises, the accounting profession has insured current and potential demand in the field of MSEs, and remains one of the most requested among all professions by micro and small enterprises
\end{abstract}

Keywords: accounting, competencies, professional skills, skills training, company, labour market professional certified public accountant, general accounting, taxation, audit, finance, educational project of the school of accounting in san marcos

* CPC. Doctor en Ciencias Contables. E-mail: afloreskonja@yahoo.es

** Economista. Magister en Educación. E-mail: hidalgoman@gmail.com 


\section{INTRODUCCIÓN}

En el marco del ejercicio profesional en un entorno cambiante, las modernas tendencias de planificación en las instituciones educativas, y los procesos de Acreditación Nacional e Internacional aplicados a las carreras profesionales, la Facultad de Ciencias Contables de la Universidad Nacional Mayor de San Marcos formuló en los años anteriores su planificación institucional y su Proyecto Educativo, que incluye su Plan de Estudios, partiendo del perfil del egresado y la definición del campo profesional. Además, la Facultad, partiendo de su autoevaluación, y de la doctrina pedagógica y de acreditación, ha dado el paso de la formulación de sus Planes de Estudios por competencias, desde 2011. Dicho Proyecto Educativo o Proyecto Curricular debe ser permanentemente revisado, a fin de contrastar su adecuación y vigencia.

A cuyo fin, es necesario contrastar permanentemente si las competencias profesionales del Contador Público Colegiado establecidos en el Proyecto Educativo o Proyecto Curricular, son los mismos que demanda el mercado laboral profesional, en sus distintos estratos o estamentos. Entre estos estamentos tenemos: la empresa privada, en sus niveles de gran empresa, mediana empresa y pequeño y microempresa; el sector público (instituciones gubernamentales), organismos no gubernamentales y demás organizaciones no empresariales. Como está claro, la empresa es la principal demandante de profesionales (seguida del Estado) y dentro de ellas, las mype constituyen un sector en ascenso, que debe ser considerado como una fuente de demanda de profesionales en ascenso también. Debe considerarse también que dichas instituciones demandantes constituyen además una parte crucial los "grupos de interés" de nuestra Facultad.

Inicialmente, en forma de un proyecto piloto, el equipo investigador del Proyecto de Investigación de título "Competencias Profesionales requeridas al Contador Público Colegiado por las Micro y pequeña empresas del Perú", presentado al Vicerrectorado de Investigación y desarrollado durante 2012, decidió formular un instrumento de recolección de información dirigido a las mype, a través de sus gremios más institucionalizados (encuesta dirigida a sus máximos directores), y conformado por preguntas acerca de:

- Las competencias profesionales del CPC más requeridas por las mype, actuales y potenciales

- Las funciones dentro de la empresa relacionadas a los CPC mas requeridas por las mype, como demanda actual y demanda potencial,

- Las características del perfil personal del profesional contable (CPC) más apreciadas,

- Demanda relativa actual y potencial de los profesionales contables respecto a las otras profesiones más demandadas.

- Tiempo y oportunidad de la demanda del profesional contable por dichas empresas.

La encuesta fue aplicada a los siete mayores y más institucionalizados gremios de las mype en el Perú, entre el 10 de septiembre y el 02 de octubre de 2012, con el valorable apoyo de un grupos de estudiantes del $4^{\circ}$ Ciclo de la Facultad, bajo la coordinación de la alumna Diana Suybate Mallma.

\section{LAS COMPETENCIAS PROFESIONALES DEL CONTADOR PÚBLICO COLEGIADO}

Las competencias, desde el punto de vista de la formación profesional, son "en sentido genérico, el conjunto de conocimientos o habilidades prácticas que otorgan capacidad de ejecución o rendimiento en una actividad determinada" (Castro Kikuchi, 2005; 132) El término competencia viene del mundo empresarial - laboral, e irrumpe en el mundo académico aparejado a un cambio de énfasis en la formación, del conocimiento impartido por el cuerpo docente y las capacidades requeridas por la actividad laboral profesional. En el caso de la educación básica, la palabra "competencia" se desliga del desempeño profesional, pero en el caso de la formación profesional, esto no es posible ni apropiado. Sin embargo, sí podemos distinguir, como hace la Facultad de Ciencias Contables y Tuning América, entre competencias genéricas, más abstractas, y las competencias específicas, que corresponden las inmediatamente a las capacidades buscadas. 
En este caso, hemos considerado las competencias específicas o capacidades definidas en la Ley 28951, Ley de Profesionalización del Contador Público, y en las definidas como competencias específicas por el Modelo Pedagógico de San Marcos, y las competencias genéricas también las hemos incluido, en referencia a las planteadas por el Plan $\mathrm{Cu}-$ rricular de la Facultad de Ciencias Contables. Esta también incluye el campo ocupacional

\section{PROYECCIONES DEL CAMPO PROFESIO- NAL DE LA CONTABILIDAD}

La carrera de Contabilidad es una de la de mayor demanda, a nivel nacional, figurando en el puesto $\mathrm{N}^{\circ} 1$ de las carreras más demandadas del ranking preparado por Piscoya Hermoza para la Asamblea Nacional de Rectores y publicado por la institución rectora del sistema universitario peruano (Piscoya, 2008; 138), seguido de Administración, Derecho y Economía Política, considerándose una profesión con futuro.

Por su relación con la tributación, aspecto ineludible para la empresa, al colaborar eficientemente en el cumplimiento de la obligación tributaria de la empresa, actividad exigida obligatoriamente por el
Estado, y que exclusivamente debe hacer el contador público, por mandato legal, el contador público tiene asegurado su campo laboral profesional, y, por el contrario, lejos de ser desplazado por otras profesiones ligadas a la empresa, como la Administración y la Economía, la Contabilidad desplaza a las demás profesiones en actividades de las ciencias de la empresa comunes a todas estas profesiones tales como la función financiera, auditoría, planificación, etc.

Como las demás profesiones, la profesión contable también está atraviesa una redefinición y una ruta de especialización, acorde con las nuevas realidades de la globalización, que acentúan aún más las competencias profesionales privativas de la profesión, y por tanto, la empleabilidad de los egresados de la carrera.

\section{PRINCIPALES RESULTADOS}

Cuando se preguntó sobre qué competencia profesionales del CPC requerirían más las mype; el de la Determinación de la Obligación Tributaria fue la primera como requerida "siempre", así como: la formulación de sistemas de costos; asesoría societal (conformación de sociedades, etc.) y asesoría financiera, aún por encima de la formulación de la Contabilidad General y los EEFF.

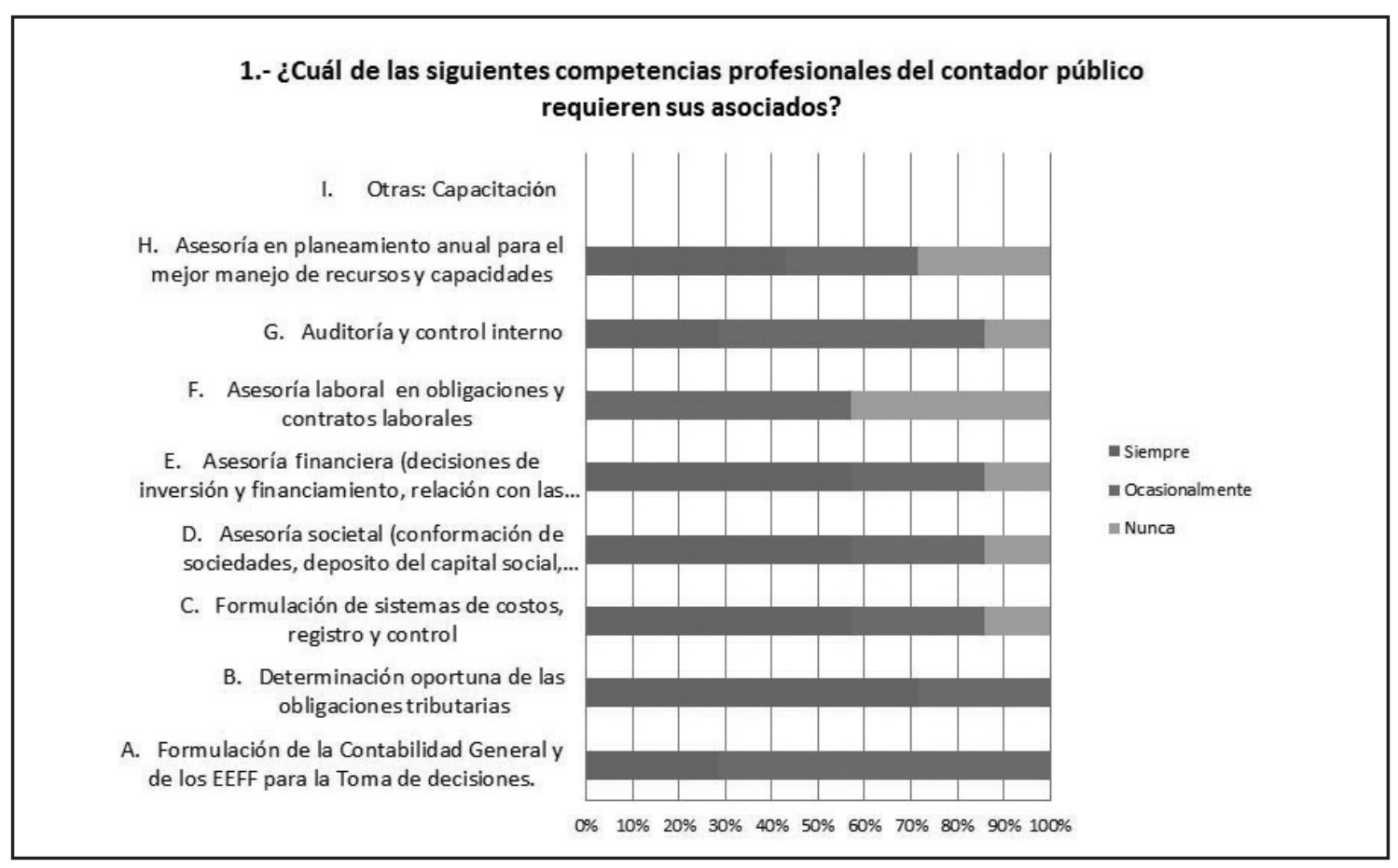


Pero además las mype consideran que estas competencias y funciones empresariales del CPC se mantendrán en el futuro, en cuyo caso las funciones de contabilidad general y formulación de los EEFF mejoran, como se nota en el cuadro siguiente:

\section{Cuadro № 2}

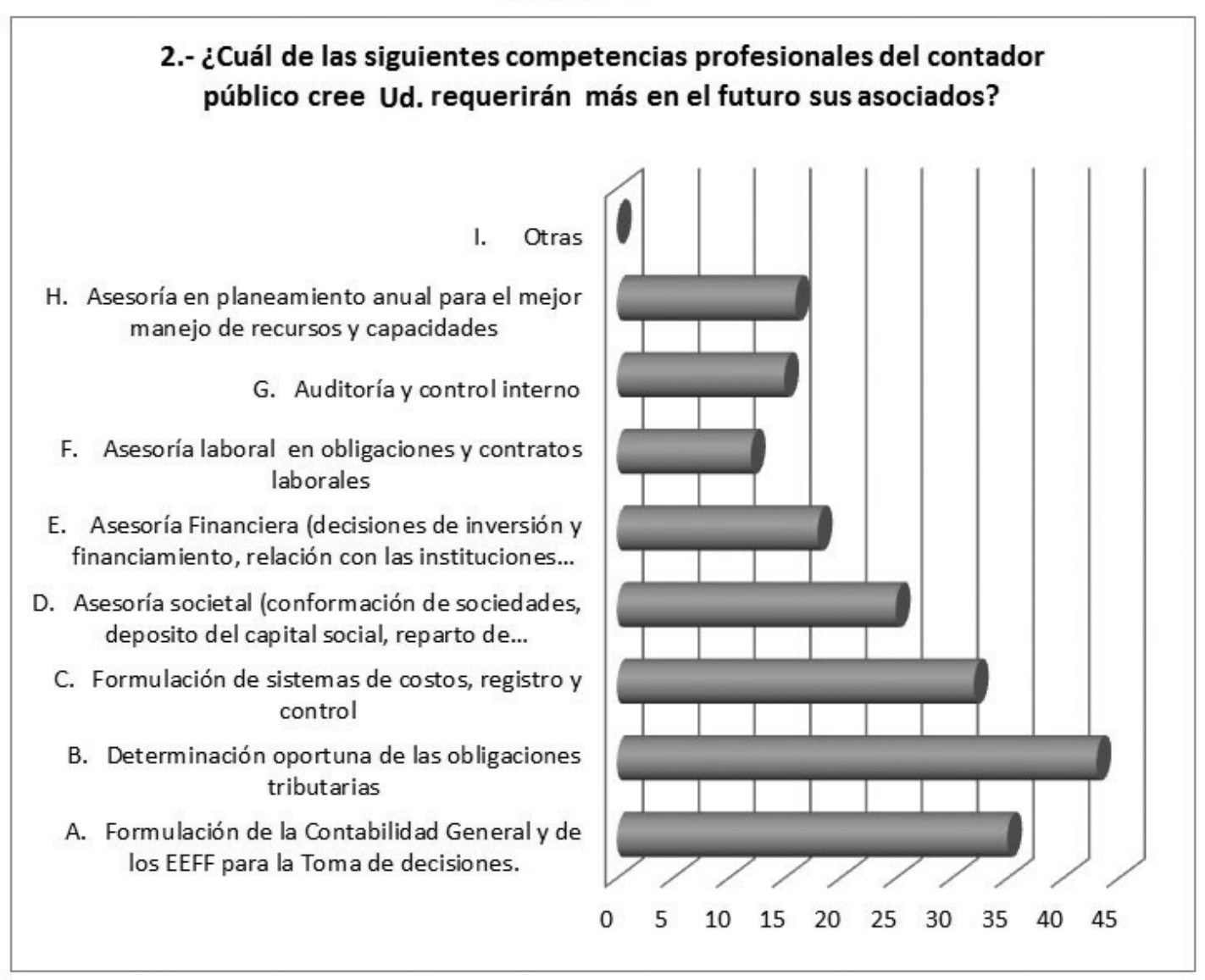

Para los líderes de las mype; Entre las habilidades personales de los CPC se destacan:

1. Análisis, investigación, razonamiento, identificación y solución de problemas en situaciones cambiantes.

2. Aptitud y disposición para trabajar en equipo.

3. Destreza para analizar riesgos y modelos de decisiones.

En el caso de las mype, caracterizadas por el tamaño de sus operaciones, en la cual los gastos administrativos no pueden ser grandes en relación al tamaño, el 57\% de los directores de las mype con- sideran que el profesional contable que se requiere más es a tiempo parcial, el 14\% a tiempo completo y el $29 \%$ opina que se requiere ocasionalmente; atribuyendo a la periodicidad de la demanda de estos servicios al nivel de ingresos; a la cultura empresarial y a las proyecciones de crecimiento de la empresa.

Respecto a la demanda futura de las carreras profesionales, se preguntó ¿Cuál de las siguientes profesiones se requerirán más en el futuro por las mype?, pidiendo se cite tres de ellas, de las cuales la Ingeniería Industrial tuvo el mayor número de citas, seguido de Contabilidad y Negocios Internacionales. 


\section{Cuadro NN 3}

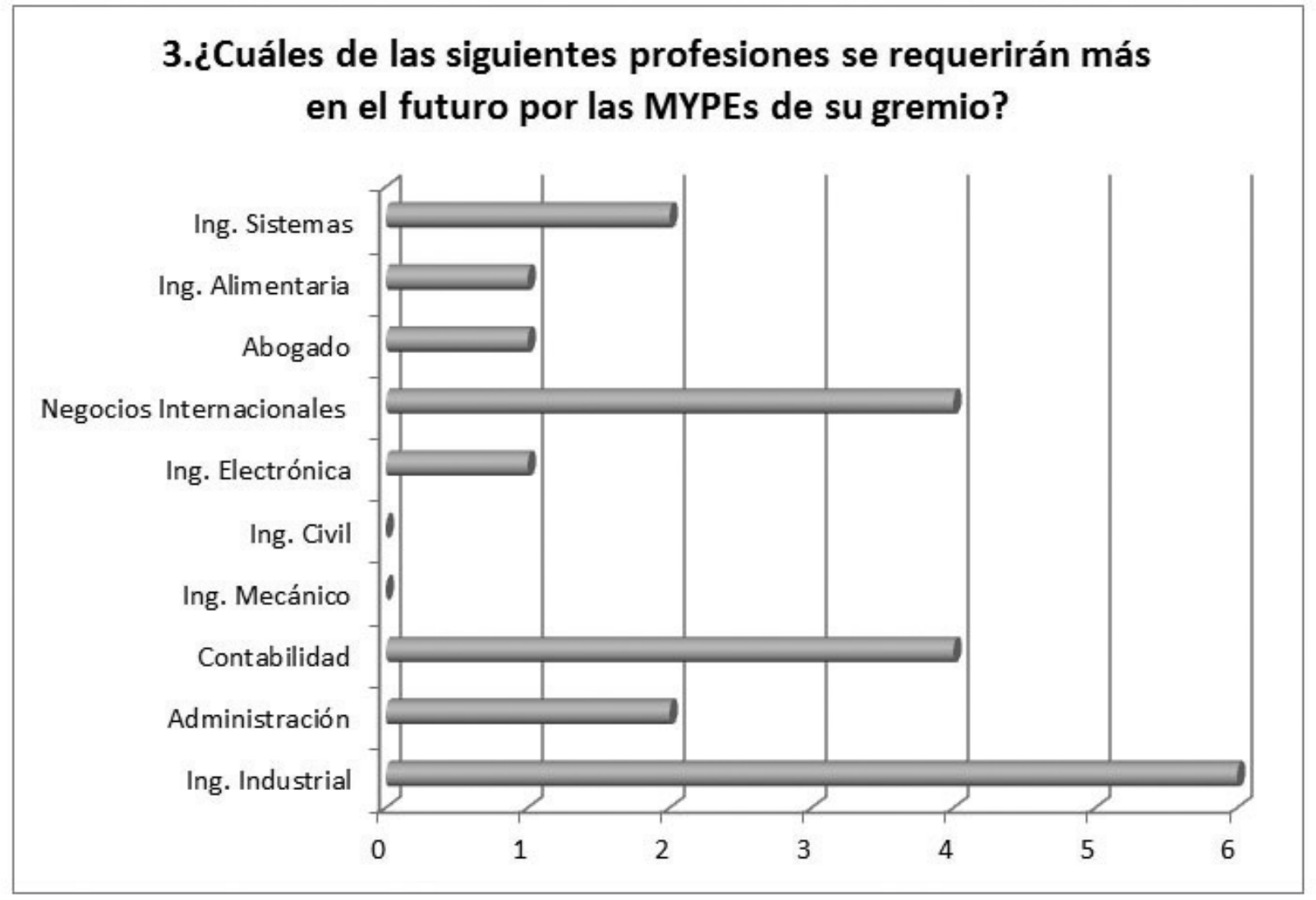

\section{CONCLUSIONES Y RECOMENDACIONES}

Las conclusiones que se obtuvieron del análisis de los resultados fueron las siguientes:

1. Las pyme requieren de los CPC principalmente para la Función Tributaria, este requerimiento y prioridad se estima se mantendrá a futuro.

2. El $57 \%$ de los líderes mype consideran que los CPC son más requeridos a Tiempo Parcial por las mype, el $14 \%$ a Tiempo Completo y el $29 \%$ Ocasionalmente.

3. Estas situaciones están determinadas principalmente por los siguientes factores: periodicidad de la demanda de estos servicios al nivel de ingresos; cultura empresarial y a las proyecciones de crecimiento de la empresa.

4. Los líderes mype consideran que las carreras de más demanda futura son: Ingeniería Industrial, Contabilidad y Negocios Internacionales.
Las recomendaciones formuladas son las siguientes:

1. Tomar en consideración las conclusiones obtenidas en la presente investigación para la revisión del Plan Curricular de las carreras de Contabilidad. Tributación y Auditoría de la FCC.

2. Tomar en consideración los resultados de la presente investigación como antecedentes para el Estudio de Demanda Social de las Carreras Profesionales de la FCC solicitado por la Acreditación Nacional.

3. Incluir a los gremios mype que han participado de la encuesta en el Comité de Involucrados (stake-holders) de Facultad de Ciencias Contables para la formulación-revisión del Plan Estratégico.

4. Ampliar la encuesta de la Encuesta para resultados que sean generalizables a todo el sector pyme y en un segundo momento al sector empresarial e institucional del conjunto.

Vol. 21(39) 2013 | QUIPURAMAYOC / 19 
Se debe revisar y ampliar el instrumento de recolección de información aplicado para futuras aplicaciones.

\section{Gremios que respondieron a la encuesta}

Nuestro agradecimiento a los directivos de los siguientes gremios:

- Asociación de Pequeños y Micro Empresarios, APEMIPE

- Programa para las pymes de Adex, PYMEADEX

- Cámara de PYMES Exportadoras del Perú

- Confederación Nacional de Gremios de la Micro y Pequeña Empresa del Perú, CONAMYPE

- Federación de Empresarios de Gamarra Perú, FEDEGA

- Registro Nacional de Micro y Pequeña Empresa, REMYPE

- Sociedad Nacional de Industrias- Comité de la Pequeña Industria

- Comité de la Pequeña Industria, COPEI

- Sociedad Regional para el Desarrollo Integral de las PYMEs, REDPYMES

\section{REFERENCIAS BIBLIOGRÁFICAS}

1. PERU. Ley Universitaria, Ley No 23733, promulgada el 09 de diciembre de 1983, publicada en el Diario Oficial El Peruano el 17 de diciembre de 1983.
2. CONEAU. CONSEJO DE EVALUACIÓN, ACREDITACIÓN Y CERTIFICACIÓN DE LA CALIDAD DE LA EDUCACIÓN SUPERIOR UNIVERSITARIA. Dirección de Evaluación y Acreditación. Modelo de Calidad para la Acreditación de Carreras Universitarias y Estándares para la Carrera de Educación. Lima 2008. Disponible en: www.oei.es/pdfs/modelo_calidad_acreditacion_universitaria.pdf

3. CONEAU. Consejo de Evaluación, Acreditación y Certificación de la Calidad de la Educación Superior Universitaria Guía para la acreditación de carreras profesionales universitarias del CONEAU El Peruano, miércoles 16 de setiembre de 2009

4. Piscoya Hermoza, Luis. Formación Universitaria vs. Mercado Laboral II Asamblea Nacional de Rectores. 2008

5. Castro Kikuchi, Luis. Diccionario de Ciencias de la Educación. Ediciones Ceguro SAC. Segunda Edición Revisada y Ampliada. Lima, Perú, 2005, p. 132

6. Beneitone. Pablo, et. al. Reflexiones y perspectivas e la Educación Superior en América Latina. In forme Final - Proyecto Tuning - America Latina 2004 - 2007. 2007. Universidad de Deusto y Universidad de Groningen. 